\title{
Uso do Arduíno como um sistema alternativo para medir radiação solar global e práticas educacionais
}

Usage of Arduino as an alternative system to measure global solar radiation and educational practices

\author{
Péricles Vale Alves*1@, Luis Henrique Souza Reis ${ }^{3}$, Carlos Alexandre S. Querino ${ }^{10}$, Marcos \\ Antônio Lima Moura ${ }^{2}$, Adalcir Araújo Feitosa Junior ${ }^{2}$, Paulo André da Silva Martins ${ }^{1}$
}

\footnotetext{
${ }^{1}$ Universidade Federal do Amazonas, Instituto de Educação, Agricultura e Ambiente, Programa de Pós-graduação em Ciências Ambientais, Humaitá, AM, Brasil

${ }^{2}$ Universidade Federal de Alagoas, Instituto de Ciências Atmosféricas, Maceió, AL, Brasil

${ }^{3}$ Universidade Federal do Amazonas, Instituto de Educação, Agricultura e Ambiente, Humaitá, AM, Brasil
}

\begin{abstract}
Recebido em 28 de outubro de 2019. Revisado em 5 de dezembro de 2019. Aceito em 7 de dezembro de 2019.
Quantificar a intensidade da radiação solar incidente na superfície terrestre é extremamente importante, pois os processos de ordem natural, sejam eles físicos, químicos e/ou biológicos dependem diretamente dela. Contudo, existem algumas dificuldades para medir esta variável, na qual destaca-se o custo elevado de equipamentos para esta finalidade. Desta forma, o objetivo deste trabalho foi desenvolver um protótipo de baixo custo para medir radiação solar global bem como promover a contextualização do ensino de física. Uma estrutura de alumínio fundido, para minimizar os efeitos térmicos e as intempéries sobre suas medidas foi desenvolvida. Esta estrutura era coberta com um difusor plano de acrílico e uma cúpula semi-esférica de vidro que envolvia o sensor fotoresistivo. O protótipo foi conectado ao arduino, usado como datalogger, através de um cabo de rede. Para a calibração do protótipo foi utilizado um piranômetro comercial modelo CM5 da KIPP \& ZONEN. Índices estatísticos para se medir a acurácia do protótipo foram aplicados. As medidas foram realizadas continuamente, a cada minuto, durante cinco dias. Os resultados mostraram: coeficiente de determinação foi de $R^{2}=0,957$, de eficiência $(\varepsilon=0,95)$, índice de concordância de Willmott $(d=0,99)$ e o erro médio absoluto $\left(E M A=51,22 \mathrm{~W} \mathrm{~m}^{-2}\right)$. Verifica-se uma boa aproximação dos valores de radiação solar global medidas pelo protótipo referentes aos do CM5. Portanto, a construção deste protótipo é útil para facilitar e difundir medidas diárias de radiação solar global e, sobre tudo, poderá contribuir com a contextualização dos conceitos estudados em Física nos ensinos básico e superior.

Palavras-chave: Sensor Fotorresistivo, Protótipo, Contextualização.
\end{abstract}

Quantifying the intensity of the incoming solar radiation at the earth's surface is important, to the natural, physical, chemical and / or biological processes. However, there are some difficulties in measuring this variable, due to the high cost of equipment for this purpose. Thus, the objective of this work was to develop a low cost prototype to measure global solar radiation. A cast aluminum frame, covered with a flat acrylic diffuser and a semi-spherical glass dome, to surround the photoresist sensor and minimize thermal and weather effects on its measurements, was made. The prototype was connected to the arduino, used as datalogger. A commercial KIPP \& ZONEN pyranometer model CM5 was used for calibration. Measurement accuracy was determined by determination coefficients $\left(R^{2}=0.957\right)$, coefficients of efficiency $(\varepsilon=0.95)$, Willmott agreement index $(d=0.99)$, and mean absolute error $\left(M A E=51.22 \mathrm{~W} \mathrm{~m}^{-2}\right)$. There is a good approximation of the global solar radiation values measured by the prototype for CM5. Therefore, the construction of this prototype is useful to facilitate and diffuse daily measurements of global solar radiation and, overall, may contribute to the contextualization of the concepts studied in physics in the highschool and colege education.

Keywords: Photoresistive Sensor, Prototype, Contextualization

\section{Introdução}

A energia produzida pelo Sol, em seu núcleo, originase da fusão entre quatro átomos de hidrogênio e um átomo de hélio é irradiada de forma contínua 11. Essa energia é transportada para as camadas mais externas do sol através de irradiação e por processos convectivos turbulentos. A partir daí, lançada para o espaço em forma

*Endereço de correspondência: periclesmat@ufam.edu.br de radiação eletromagnética abrangendo, praticamente, todos os intervalos do espectro eletromagnético, desde os raios gama às ondas de rádio até atingir a superfície da terra $[2]$.

Assim, quantificar a intensidade da radiação solar na superfície terrestre é de fundamental importância, pois todos os processos de ordem natural, sejam eles físicos, químicos e/ou biológicos, necessitam da radiação solar 3 5. Alguns dos processos mais importantes são: 
- a fotossíntese, que é fundamental para o desenvolvimento de plantas;

- o aquecimento do planeta, que mantém uma temperatura ideal para que haja vida, através do efeito estufa;

- o corpo humano que necessita de raios solares na síntese de nutrientes como a vitamina D, que ajuda na fixação do cálcio;

- além do aproveitamento dessa energia para a transformação em energia elétrica, considerada atualmente uma fonte de energia limpa.

Entretanto, no Brasil, as medidas da radiação eletromagnética à superfície são recentes e possuem poucos dados históricos. Em geral, esses dados são produzidos por Universidades e Centros de Pesquisa onde se destacam Instituto Nacional de Pesquisas Espaciais (INPE), o Instituto Nacional de Meteorologia (INMET) e a Embrapa.

O custo elevado de equipamentos para medir a radiação solar em superfície, como os piranômetros, torna escasso os dados referentes a irradiância solar, restringindo o acesso à informações desta variável para determinadas localidades do país como, por exemplo, na região norte do Brasil.

Assim, a construção de um protótipo de baixo custo, que estime valores fidedignos de radiação solar global, possibilitam potencializar laudos climáticos de uma determinada região, além de ser uma temática que tem ganho atenção no meio científico [6].

Além de todos os fatores já mencionados anteriormente, este trabalho promove a contextualização de temas relacionados à física, desde a etapa de construção do protótipo à coleta de dados.

De acordo com a Base Nacional Comum Curricular - BNCC, contextualizar os conteúdos dos componentes curriculares, identificando estratégias para apresentá-los, exemplificá-los, conectá-los e torná-los significativos, com base na realidade do lugar e do tempo nos quais as aprendizagens estão situadas [7].

Assim, o professor pode, por exemplo, explorar alguns conceitos abordados no ensino de física, tais como: circuitos elétricos e resistores que agrega em sua montagem uma gama de componentes, como resistores ôhmicos e não ôhmicos, e posicionamento dos mesmos quanto as suas associações (série ou paralelo) 8]. Logo, possibilita ser discutida a temática de forma prática em sala de aula com o auxílio do protótipo Fig. (1);

As ópticas geométrica e ondulatória no qual originou a Lei da refração (Eq. 1) elaborada por Willebrord Snell que, posteriormente (1637), foi ratificada por Descartes que afirmou: um raio refratado também permanece no plano de incidência:

$$
\eta_{12}=\frac{\sin \theta_{1}}{\sin \theta_{2}}
$$

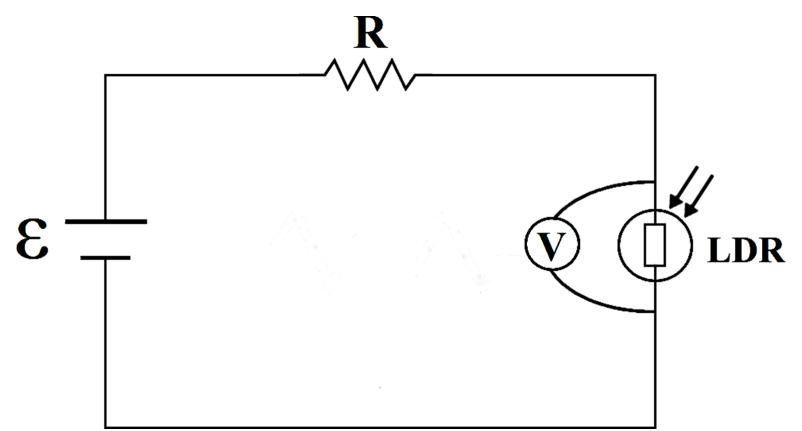

Figura 1: Esboço do circuito usado no projeto, em que $\varepsilon$ é a tensão de entrada, $R$ é o resistor, $V$ representa o sensor de tensão e $L D R$ é o fotosensor de resistência variável. Fonte: acervo dos autores.

onde a constante ${ }^{1} \eta_{12}$ é conhecida por índice de refringência do meio 2 com relação ao meio 1 .

Se a luz, ao atravessar do meio 1 para o meio 2 resultar em $\eta_{12}>1$, diz-se que o meio 2 é mais refringente e o raio refratado se aproxima da normal, ou se $\eta_{12}<1$, diz-se que o meio 2 é menos refringente e o raio refratado se afasta da normal [9]; dispersão da luz que é um fenômeno óptico responsável pela separação das cores que constituem a luz branca e pode ser observado em um prisma de vidro ou em fenômenos naturais como o arco-íris 9].

$\mathrm{O}$ célebre físico e matemático, Isaac Newton, verificou este fenômeno no ano de 1672 e publicou um trabalho, no qual apresentava suas ideias sobre a natureza das cores.

Desta forma, este trabalho teve por objetivo apresentar a construção de um piranômetro de baixo custo para estimar valores de radiação solar global diária bem como promover a contextualização do ensino de física.

\section{Materiais e métodos}

\subsection{Construção do protótipo}

Foi utilizado um dispositivo fotorresitivo (LDR ${ }^{2}$ (Fig. 2 produzido a partir de um material semicondutor, cuja resistência varia de forma quase linear com a variação da intensidade de luz incidente, além de ser um dispositivo não polarizado cuja corrente pode circular em ambos os sentidos 10].

Dois pistões usados, de motor a diesel, adaptado em uma tornearia, foram utilizados como um corpo protetor e, posteriormente, acoplados ao LDR.(Fig. 3 ).

Acima do LDR foi acoplado o difusor, com $4 \mathrm{~mm}$ de espessura e $3 \mathrm{~cm}$ de diâmetro, feito em material acrílico contendo duas partes, uma translucida e outra transparente (Fig. 4).

O circuito elétrico foi construído com um cabo de rede para possibilitar o envio do sinal até a plataforma arduíno. Os demais componentes como o resistor $(100 \mathrm{~K} \Omega)$, a fonte

\footnotetext{
${ }^{1}$ Válida para luz monocromática, pois varia com de uma cor para outra.

${ }^{2}$ Light Dependent Resistor
} 


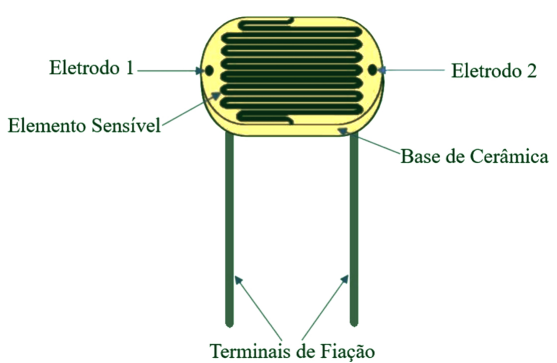

Figura 2: Fotoresistor também conhecido como LDR (Resistor Dependente de Luz). Fonte: acervo dos autores.

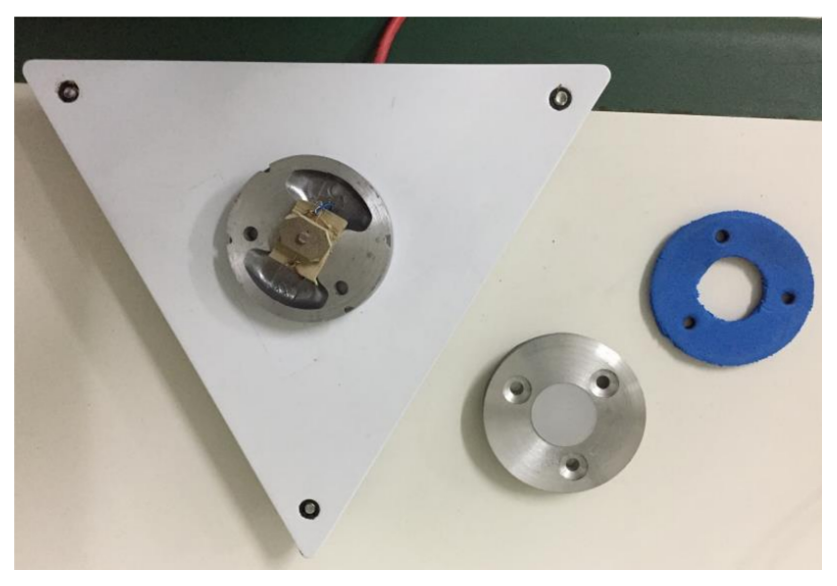

Figura 3: Carcaça dos pistões depois de moldados na tornearia e com os furos para encaixe da parte superior à base. Fonte: fotos dos autores.

de alimetação contínua de 5V, nobreak (para falta de energia e estabilizar a tensão fornecida) e um kit arduino uno foram instalados no interior de uma sala, e ficaram inteiramente protegidos das intemperes climáticas.

Para maior proteção das partes internas e evitar condições adversas, foi colocado na parte superior do protótipo uma cúpula de vidro com forma semiesférica e resistente ao calor visto na Fig. (5).

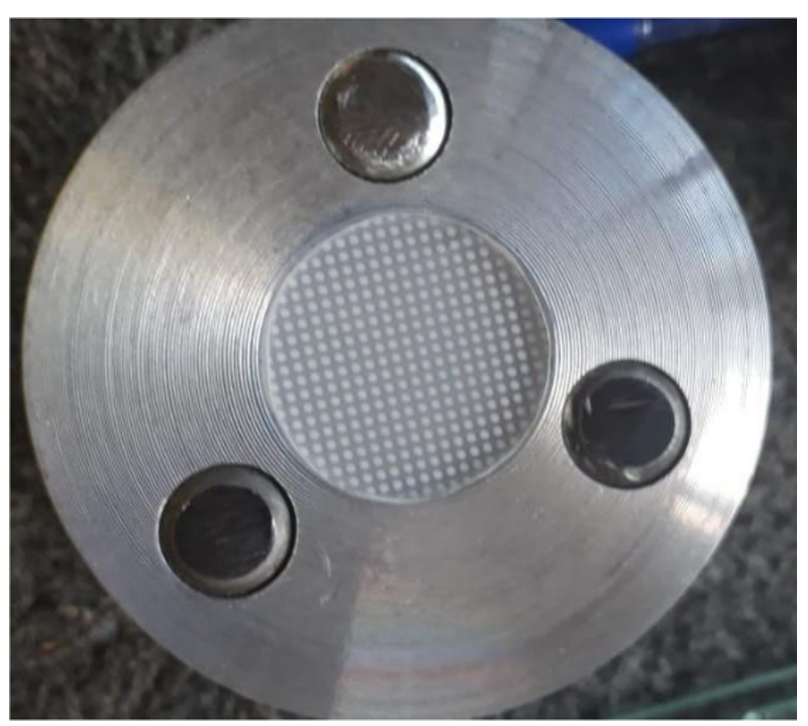

Figura 4: Protótipo com o material difusor. Fonte: fotos dos autores.

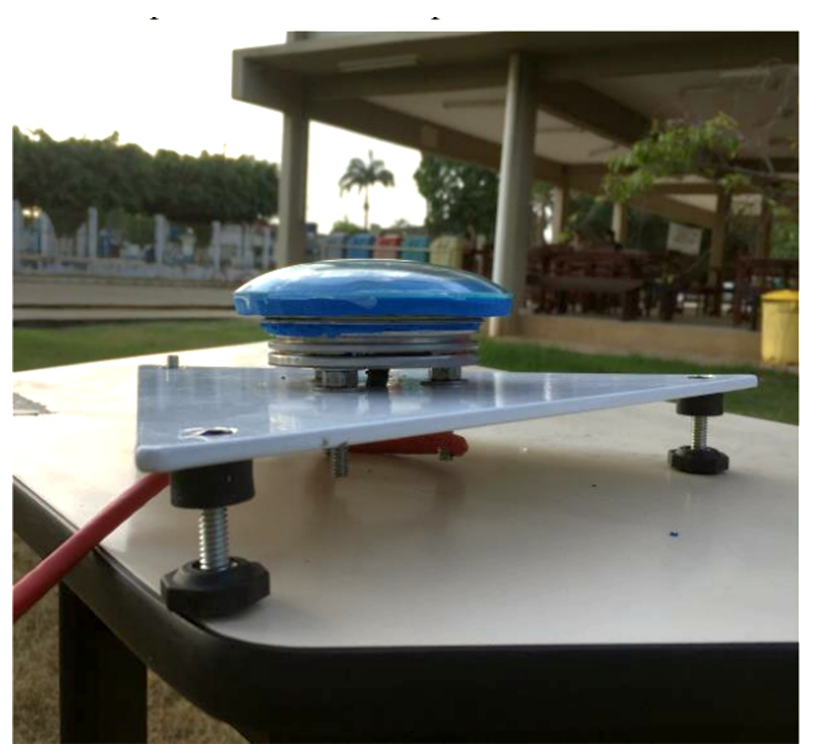

Figura 5: Protótipo estruturado e conectado via cabo de rede a plataforma arduino para a coleta de dados. Fonte: foto dos autores.

Tabela 1: Descrição e valores dos materiais utilizados para construção do protótipo para medir a radiação solar global.

\begin{tabular}{lclc}
\hline Materiais & Quantidade & Especificações & Valor $(\mathrm{R} \$)$ \\
\hline LDR $^{*}$ & 1 & CdS & 5,60 \\
pistões usados* $^{*}$ & 2 & Motor à diesel & 30,0 \\
parafusos* & 3 & $8,0 \mathrm{~cm}$ & 3,00 \\
Cúpula de vidro* & 1 & Vidro de relógio & 7,00 \\
Difusor* & 1 & Acrílico & 0,00 \\
Cabo de rede* & 1 & $10 \mathrm{~m}$ & 7,80 \\
Arduino Uno R3* & 1 & Data Logger & 35,00 \\
Resistor ôhmico* & 1 & $100 \mathrm{k} \Omega$ & 1,20 \\
Fonte DC* & 1 & $5 \mathrm{~V}$ & 10,0 \\
Nobreak & 1 & $1200 \mathrm{VA}$ & 459,00 \\
\hline
\end{tabular}

*Materiais indispensáveis para construção do protótipo. 


\subsection{Coleta e tratamento dos dados}

\subsubsection{Coleta de dados}

O protótipo foi instalado ao lado de um piranômetro, modelo CM5 (Kipp \& Zonen, Depht, Holanda) cuja constante de calibração é $89,10818 \mathrm{~W} \mathrm{~m}^{-2} \cdot \mathrm{mV}^{-1}$. Ambos sensores foram deixados ao ar livre sobre uma superfície plana e horizontal, durante cinco dias, no alto do Bloco 2 do Instituto de Educação, Agricultura e Ambiente,

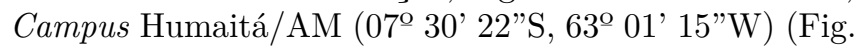
6). Ressalta-se que os dados foram coletados em horário local (-4h em relação a Greenwich).

As leituras de tensão $(\mathrm{mV})$ a cada minuto, foram realizadas por meio de uma programação simples na plataforma arduino e, posteriormente, armazenadas em um módulo $S D$. Ressalta-se que, as leituras do CM5 foram realizadas num intervalo de tempo de 10 s e as médias armazenadas a cada dez minutos em um datalogger, marca Campbell Sci modelo CR10. Assim, foi necessário fazer a média dos valores do protótipo para este mesmo intervalo.

As observações foram realizadas nos dias 04, 05, 09, 10 e 15 de outubro de 2018, com condições de nebulosidades distintas.

\subsubsection{Tratamento de dados}

Os valores de radiação solar a partir do protótipo foram estimados a partir de correlações horárias e diárias com os valores de radiação $\left(\mathrm{W} \mathrm{m}^{-2}\right)$ do CM5 com os valores de tensão $(\mathrm{mV})$ do protótipo para cada dia de teste. Desta forma, obteve-se para cada dia de teste uma equação que correlaciona a radiação solar global em função da tensão. Os dados observados, em ambos o sensores, foram plotados em gráficos, proporcionando uma melhor visualização e interpretação dos mesmos.

A acurácia da radiação solar global medida pelo protótipo, foi determinada por índices estatísticos, tais como:

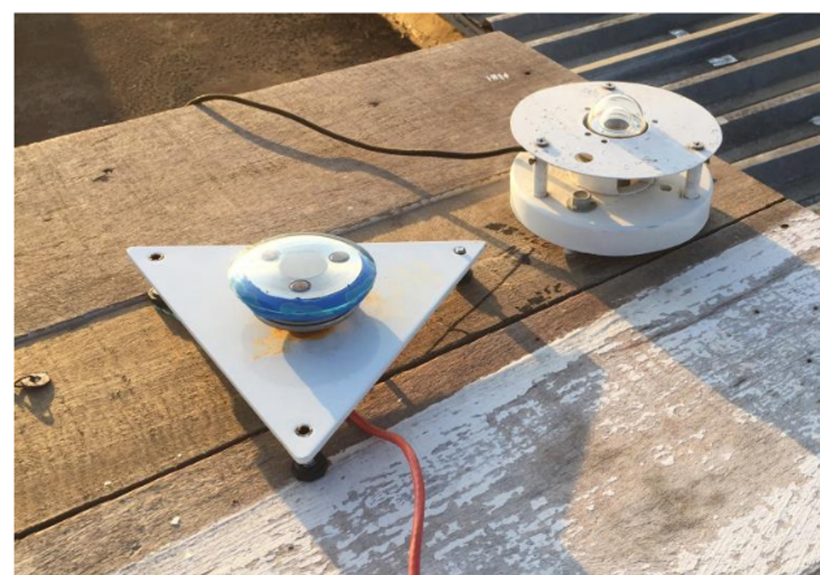

Figura 6: Protótipo colocado ao lado do piranômetro de referência, ambos nas mesmas condições climáticas, para a coleta de dados. Fonte: foto dos autores. coeficiente de determinação (Equação 2), coeficiente de eficiência (Equação 3), Índice de concordância de Willmott (Equação 4 ) e o Erro Médio Absoluto (Equação 5.

$$
\begin{gathered}
R^{2}=\frac{\left[\sum_{i=1}^{n}\left(O_{i}-\bar{O}\right)\left(P_{i}-\bar{P}\right)\right]^{2}}{\sum_{i=1}^{n}\left(O_{i}-\bar{O}\right)^{2} \sum_{i=1}^{n}\left(P_{i}-\bar{P}\right)^{2}} . \\
\varepsilon=1-\frac{\sum_{i=1}^{n}\left(O_{i}-P_{i}\right)^{2}}{\sum_{i=1}^{n}\left(O_{i}-\bar{O}\right)^{2}} . \\
d=1-\frac{\sum_{i=1}^{n}\left(O_{i}-P_{i}\right)^{2}}{\sum_{i=1}^{n}\left(\left|P_{i}-\bar{O}\right|+\left|O_{i}-\bar{O}\right|\right)^{2}} . \\
E M A=\frac{1}{n} \sum_{i=1}^{n}\left|O_{i}-P_{i}\right| .
\end{gathered}
$$

O coeficiente de determinação (Equação 2) descreve a fração da variância total nos dados observados ou o quanto os dados observados podem ser explicados pelo modelo [11].

A Equação 3 é o coeficiente de eficiência (também chamado de coeficiente de Nash-Sutcliffe) que determina a magnitude relativa da variância residual do modelo em comparação com a variância dos dados medidos [12].

O índice de concordância de Willmott (Equação 4) permite avaliar desvios entre os valores da radiação solar global observados pelo CM5 e estimados pelo protótipo, tendo seu valor variando de 0 a 1 . Quanto menor o desvio, melhor o desempenho da metodologia avaliada 12 .

A Equação 5 é o Erro Médio Absoluto o qual não leva em consideração se um erro foi superestimado ou subestimado e, caracteriza-se por ser a média dos erros cometidos pelo modelo de previsão durante uma série para um determinado período [11.

Importante frisar que, nas Equações (2), (3), (4) e (5), $O_{i}$ são os dados observados no CM5, $P_{i}$ são os dados preditos pelo protótipo, e que $\bar{O}$ e $\bar{P}$ são as médias da radiação solar medidas no CM5 e no protótipo, respectivamente, em todo o período de aquisição de dados.

\section{Resultados e discussões}

\subsection{Análise horária do protótipo}

A relação dos valores de radiação solar global $\left(R_{g}\right)$ medidas pelo CM5, com os valores de tensão, $\Delta V$, medidas 
pelo protótipo, ajustam-se melhor com a curva exponencial, ou seja, podemos associar para cada dia de teste, uma equação da forma:

$$
R_{g}(\Delta V)=\alpha e^{\beta \Delta V}
$$

em que $\alpha$ e $\beta$ são constantes, cujos valores são apresentados na Tab. (2).

A não linearidade da Equação (6) se dá pelo fato de o LDR não exibir uma resposta linear quando submetido a variação da intensidade luminosa. Assim, esta equação indica uma assinatura precisa do comportamento do sensor escolhido. Então, tomando-se a média para cada constante (Tab. 2] 13], obtém-se uma equação que relaciona $R_{g}$ e $\Delta V$ dada por:

$$
R_{g}(\Delta V)=3,92825 e^{0,00141 \Delta V}
$$

A partir da Equação (7) pode-se realizar estimativas de $R_{g}$ em função do $\Delta V$ medido pelo protótipo.

Para avaliar o desempenho do protótipo baseado na Equação (7), observa-se o seu comportamento horário

Tabela 2: Valores das constantes $\left(\alpha ; \mathrm{W} \mathrm{m}{ }^{-2}\right)$ e $\left(\beta ; \mathrm{mV}^{-1}\right)$ obtidos a partir da comparação entre $\Delta V$ e $R_{g}$ para cada dia de teste.

\begin{tabular}{ccc}
\hline Dia de teste & $\alpha\left(\mathrm{W} \mathrm{m}^{-2}\right)$ & $\beta(\mathrm{mV})^{-1}$ \\
\hline $04 / 10 / 2018$ & 2,75798 & 0,00148 \\
$05 / 10 / 2018$ & 4,34082 & 0,00138 \\
$09 / 10 / 2018$ & 3,99054 & 0,00142 \\
$10 / 10 / 2018$ & 4,74821 & 0,00136 \\
$15 / 10 / 2018$ & 3,80371 & 0,00144 \\
\hline MÉDIA & 3,92825 & 0,00141 \\
\hline
\end{tabular}

quando comparado com o piranômetro CM5. A Fig. 77 mostra que os valores horários preditos pelo protótipo não são, necessariamente, fidedignos quando comparados ao CM5.

Do amanhecer até próximo ao ápice da irradiação solar à superfície terrestre (às $12 \mathrm{~h}$ ), o protótipo tende a superestimar os valores observados no CM5. Por outro lado, em todos os dias de observação, entre às $12 \mathrm{~h}$ até o pôr do sol, é predominante que o protótipo subestime os valores, com raras observações onde ele superestima ou é congruente aos valores observados pelo CM5.

Esse comportamento horário irregular do protótipo está associado a dois fatores:

\section{i) a limitação espectral do sensor LDR}

O LDR utilizado possui resposta espectral máxima de $540 \mathrm{~nm}$ na sua sensibilidade máxima à luz. Os valores instantâneos da radiação solar global à superfície, sofrem grandes variações temporais e espaciais. Em geral, essas variações estão em função das condições atmosféricas, especialmente umidade e nebulosidade, além da época do ano e hora do dia 4].

Além disso, ocorre o espalhamento das ondas eletromagnéticas na atmosfera e está associado, em grande parte, ao tamanho das moléculas de gás ou aerossóis presentes na atmosfera. Tal espalhamento, explica o azul do céu e o avermelhado do por do sol e é conhecido por espalhamento Rayleigh 14 .

O espalhamento Rayleigh demonstra que é a atmosfera terrestre quem espalha a luz solar e, como o azul tem comprimento de onda, $\lambda$, menor que o do vermelho, ele é mais espalhado que este, chegando aos olhos humanos com maior intensidade. Já no pôr do sol, a camada de ar que fica entre a superfície terrestre e o sol é muito

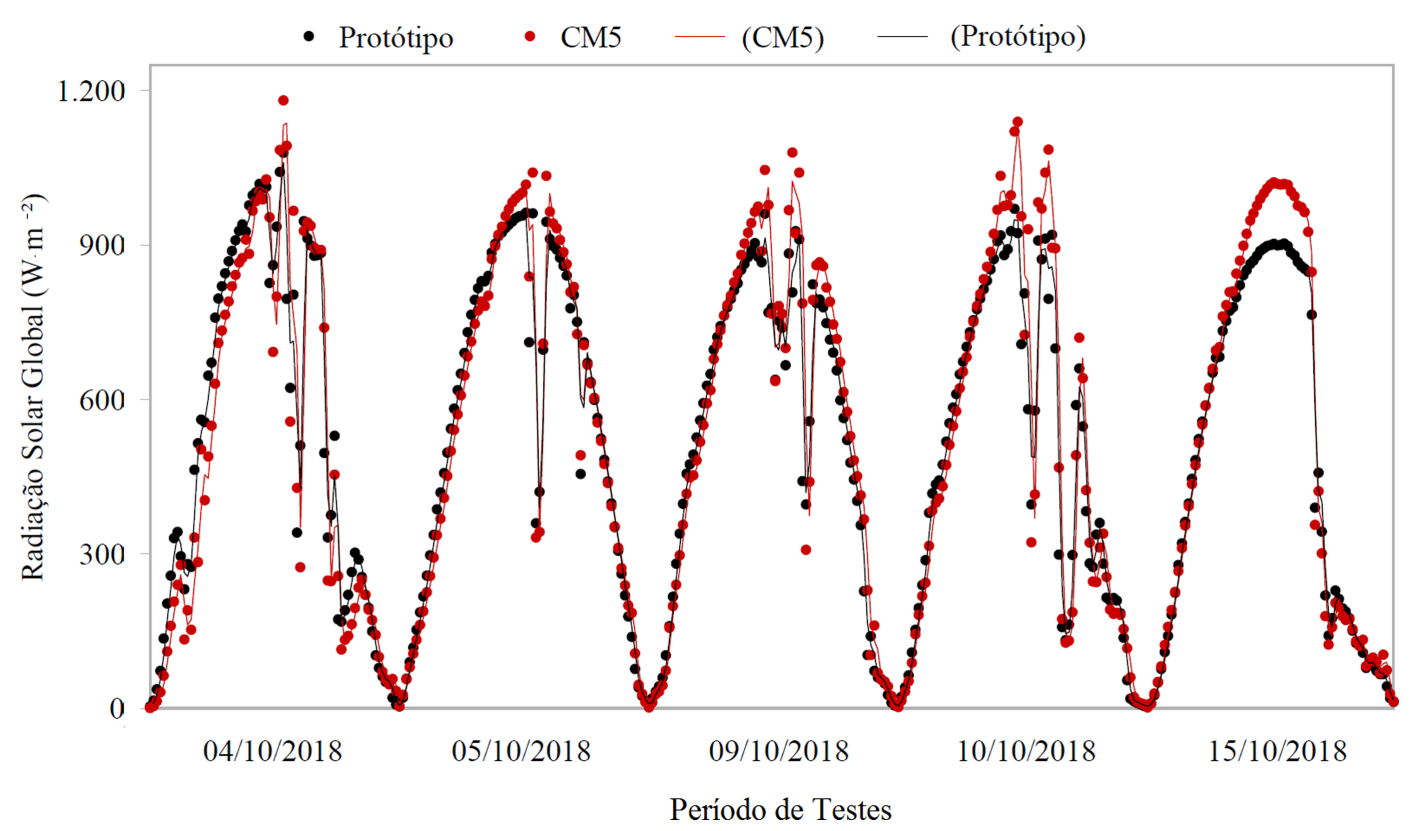

Figura 7: Comparação horária entre os valores de radiação solar global medidas no protótipo e no CM5 do nascer ao pôr do sol. Fonte: Dados dos autores. 
mais espessa. Logo, o azul é tão espalhado que além de sair da linha visão do olho humano é praticamente atenuado por completo e, o observador passa a receber maior intensidade de vermelho.

Assim, este espalhamento contribui na defasagem das medidas horárias obtidas pelo protótipo, visto que a faixa espectral de trabalho do LDR se assemelha ao comportamento do olho humano, porém limitado a um $\lambda$ abaixo do vermelho. Logo, isto explica porque o protótipo tende a subestimar os valores de radiação após às $12 \mathrm{~h}$.

Uma proposta didática simplória para ser aplicada no Ensino Médio, construída com o objetivo de mostrar o espalhamento da intensidade da luz por pequenas partículas na atmosfera, o que explica o porquê do azul do céu e o vermelho do pôr-do-sol, pode ser vista no trabalho 15 .

\section{ii) tempo de resposta lenta do LDR}

Os LDRs são dispositivos de resposta lenta no que se refere à diminuição ou aumento da resistência que, por sua vez, se relaciona ao aumento, diminuição ou corte da incidência da luz. Na realidade, essas duas respostas se apresentam de formas distintas (Fig. 8) onde o aumento de tensão no circuito é mais lento do que a queda.

Assim, os valores superestimados entre as $12 \mathrm{~h}$ e $16 \mathrm{~h}$ que, por vezes, aparecem na Fig. (7), são compreendidos pela interferência de nuvens no caminho óptico da luz até os sensores, fazendo com que está limitação reflita nos valores medidos.

Ainda que o protótipo possua as limitações apresentadas, sua utilização para a finalidade proposta não é descartada. Para garantir de fato seu uso, será feito a seguir, com objetivo de otimizar o desempenho do protótipo, a análise de parâmetros estatísticos [12 para quantificar a semelhança entre o CM5 e protótipo.

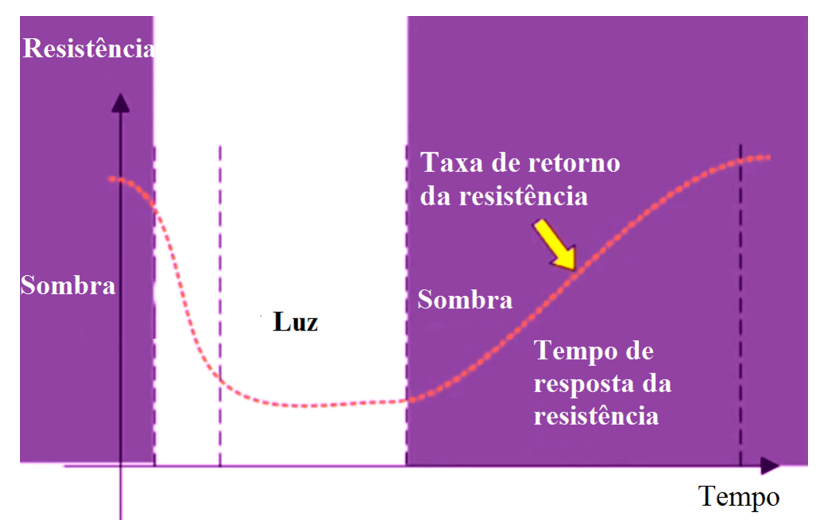

Figura 8: Gráfico representativo da variação da resistência de um LDR pelo tempo de resposta com a variação da intensidade luminosa. Fonte: Adaptada da ref. [16].

\subsection{Análise estatística}

O número amostral de $N=365$ dados foi observado no período de cinco dias de testes, ou seja, em média 73 medidas realizadas por dia.

A Equação (7) parametriza os valores de $R_{g}$ a partir dos valores de $\Delta V$, medidos no protótipo. A Tab. (3) apresenta os índices estatísticos que mostra que o desempenho do protótipo no período indicado é satisfatório.

Nesse estudo, o protótipo apresentou desempenho de $96 \%$ quando comparado os valores estimados pela Equação (7) com os esperados para o período medidos pelo CM5 (Fig. 9). Logo, verifica-se que o modelo proposto na Equação (7) indica que o protótipo exibe valores satisfatórios para o período, pois o $\varepsilon$ encontrado está próximo de 1 (Tab. 3) assim como para o coeficiente $d$, ou seja, o protótipo desempenha seu papel com excelente confiança, mostrando que os resultados obtidos pelo protótipo quando comparados aos do CM5, são excelentes.

Por fim, o valor obtido para EMA no período de estudo foi de $51,22 \mathrm{~W} \mathrm{~m}^{-2}$ (Tab. 3), ou seja, menor que $10 \mathrm{~W}$ $\mathrm{m}^{-2} \mathrm{dia}^{-1}$ o que nos permite afirmar que o desempenho do protótipo é, de fato, satisfatório.

\section{Conclusão}

O protótipo proposto neste trabalho, apresentou um comportamento irregular para dados horários devido as limitações espectrais do sensor escolhido. Por outro lado, excelentes resultados foram obtidos, quando observado um conjunto de dados referentes aos cinco dias de teste.

É evidente que se espera de um sensor a mesma acurácia para dados horários e/ou para uma série de dados mais robusta. No entanto, levando-se em consideração que foi empregado cerca de $\mathrm{R} \$ 100,00$ com materiais indispensáveis para a construção deste protótipo, tem-se, portanto, um sensor que pode ser empregado para fazer estimativa de radiação solar global para períodos de cinco em cinco dias.

No que diz respeito a contextualização para o ensino da física a montagem do protótipo deixa claro diversos campos que podem ser explorados como circuitos elétrico, óptica física e geométrica além de conceitos de física moderna. Dessa forma, esse experimento é indicado para aulas práticas tanto no ensino médio como no nível superior.

Tabela 3: Índices estatísticos obtidos, com as médias dos 05 (cinco) dias, através da correlação entre os valores medidos pelo CM5 e a equação (7).

\begin{tabular}{ccc}
\hline Símbolos & Índices & Valor \\
\hline$R^{2}$ & Coef. de determinação & 0,96 \\
$\varepsilon$ & Coef. de eficiência & 0,95 \\
$d$ & Concordância de Willmott & 0,99 \\
$E M A\left(\mathrm{~W} \mathrm{~m}^{-2}\right)$ & Erro médio absoluto & 51,22 \\
\hline
\end{tabular}




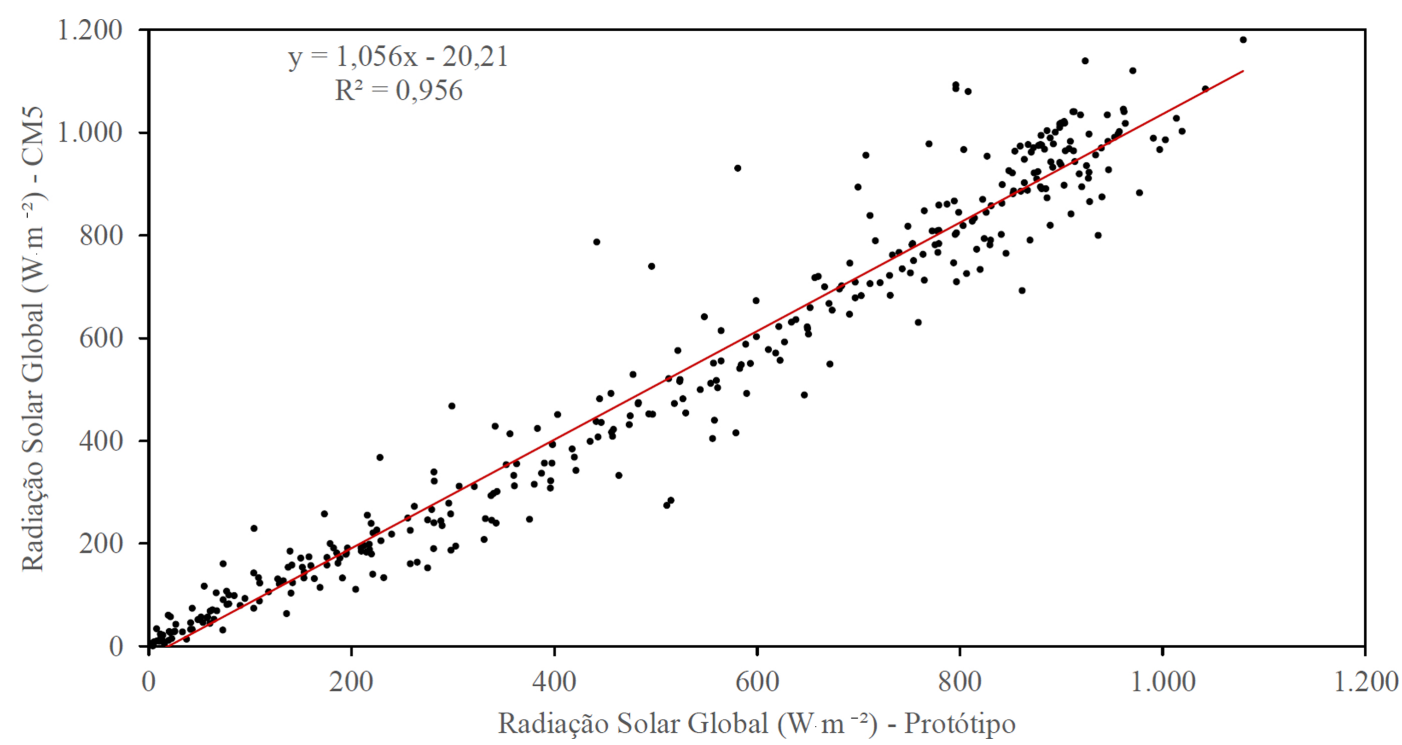

Figura 9: Gráfico da correlação linear entre os valores da radiação solar global do CM5 com os preditos pelo protótipo. Fonte: dados dos autores.

\section{Agradecimentos}

Gostaríamos de agradecer ao Prof. Dr. Marcos Moura do Departamento de Ciências Atmosféricas da Universidade Federal de Alagoas que, gentilmente, cedeu o piranômetro para que pudéssemos calibrar nosso protótipo.

\section{Referências}

[1] K. Sakurai, Space Science Reviews 38, 243 (1984).

[2] J.M. Rodríguez Gómez, F. Carlesso, L.E. Vieira e L. Silva, Revista Brasileira de Ensino de Física 40, e3312 (2018).

[3] C.A.S. Querino, M.A.L. Moura, J.K.A.S. Querino, C. Von Radow e A. Marques Filho, Revista Brasileira de Meteorologia 26, 204 (2011).

[4] C.A.S. Querino, M.A.L. Moura, R.F. Lyra and G.L. Mariano, Revista Brasileira de Meteorologia 21, 42 (2006).

[5] F.R. Martins, E.B. Pereira e M.P.S. Echer, Revista Brasileira de Ensino de Física 26, 145 (2004).

[6] A. Silva Filho, M. Biudes, N. Machado, C.A. Querino e E.S. Almeida Júnior, Revista Brasileira de Climatologia 24, 134 (2019).

[7] P.S. Marchelli, Revista de Estudos de Cultura 7, 53 (2017).

[8] H.M. Nussenzveig, Curso de física básica: Eletromagnetismo (Editora Blucher, São Paulo, 1997), v. 3.

[9] H.M. Nussenzveig, Curso de física básica (Editora Blucher, São Paulo, 2002), v. 4.

[10] H.G. Gutierre, M.S. Ribeiro, L.A.A. Pereira, G.K. Cruz, R.F. Turchiello e S.L. Gómez, Revista Brasileira de Ensino de Física 39, e3501 (2017).

[11] A. Santoro, V. Oguri e J.R. Mahon, Estimativas e erros em experimentos de física (Editora da Universidade do Estado do Rio de Janeiro, Rio de Janeiro, 2008).

[12] D.R. Legates e G.J. McCabe, Water resources research 35, 233 (1999).
[13] A.P. Oliveira, J.F. Escobedo, A. Plana-Fattori, J. Soares e P.M. Santos, Revista Brasileira de Geofísica 14, 203 (1996).

[14] R.M. Eisberg e R. Resnick. Fisica quantica: atomos, moleculas, solidos, nucleos e particulas (Editora Campus, São Paulo, 1994).

[15] M.N. Rocha, T.G. Fujimoto, R.S. Azevedo e M. Muramatsu, Revista Brasileira de Ensino de Física 32, 3501 (2010).

[16] https://youtu.be/ilN8XIK77dc, acessado em $12 / 05 / 2019$. 